\title{
HALF-ISOMORPHISMS OF FINITE AUTOMORPHIC MOUFANG LOOPS.
}

\author{
A. GRISHKOV, M.L.MERLINI GIULIANI, M. RASSKAZOVA, \\ AND L. SABININA
}

\begin{abstract}
We show that each half-automorphism of a finite automorphic Moufang loop is trivial. In general this is not true for finite left automorphic Moufang loops and for finite automorphic loops.
\end{abstract}

\section{INTRODUCTION.}

A half-isomorphism of a loop is bijection $\tau$ such that $\tau(x y)$ equals either $\tau(x) \tau(y)$ or $\tau(y) \tau(x)$ for all elements $x, y$. For groups all halfisomorphisms are either an isomorphism or an anti-isomorphism as was shown by Scott [18]. Following the terminology of [18] we will call such half-isomorphisms trivial. In general for loops the situation is different: there exist Moufang loops with half-isomorphisms which are neither an isomorphism nor an anti-isomorphism. In many cases, in particular, for finite Moufang loops of odd order, all half-isomorphisms are trivial ([5],[15]). Obviously the half-isomorphisms of a loop always form a group, which is a new and, at the time of the writing this note, rather mysterious invariant of a loop. Let us note that any anti-isomorphism of a Moufang loop $M$ to a Moufang loop $M_{1}$ is the composition of an isomorphism of $M$ to $M_{1}$ and inverse map on $M_{1}$.

\section{Preliminaries And Definitions.}

In this paper we consider a loop as a universal algebra $\langle Q ; \cdot, \backslash, /, 1\rangle$ of type $(3,0,1)$ such that the identities

$$
\begin{gathered}
(x \cdot y) / y=x=(x / y) \cdot y, \\
x \cdot(x \backslash y)=y=x \backslash(x \cdot y), \\
x \cdot 1=x=1 \cdot x
\end{gathered}
$$

hold for all $x, y \in L$. In the following we often write $x y$ instead of $x \cdot y$. 
A Moufang loop is a loop in which one of the following equivalent identities holds:

$$
\begin{aligned}
& ((x y) x) z=x(y(x z)) \\
& ((x y) z) y=x(y(z y)), \\
& (x y)(z x)=(x(y z)) x .
\end{aligned}
$$

The bijection $L_{a}: Q \rightarrow Q, L_{a} x=a x$ is called a left multiplication, analogously the bijection $R_{a}: Q \rightarrow Q, R_{a} y=y a$ is called a right multiplication for all $a \in Q$.

With an arbitrary loop $Q$ one may associate several groups of transformations of the set $Q$, such as:

- the multiplication group $\operatorname{Mlt}(Q)$ generated by the left and right multiplications by elements of $Q$;

- the left multiplication group $\operatorname{LMlt}(Q) \subseteq \operatorname{Mlt}(Q)$ generated by the left multiplications only;

- the inner mapping group $\operatorname{Inn}(Q) \subseteq \operatorname{Mlt}(\mathrm{Q})$ defined as the stabilizer of the neutral element $1 \in Q$;

- the left inner mapping group $\operatorname{LInn}(Q)$ which is the stabilizer of 1 in $\operatorname{LMlt}(Q)$.

$\operatorname{Inn}(Q)$, the inner mapping group of $Q$, is generated by three families of elements of $\operatorname{Mlt}(\mathrm{Q})$ :

$$
\begin{gathered}
\ell_{x, y}=L_{x y}^{-1} \circ L_{x} \circ L_{y}, \\
r_{x, y}=R_{x y}^{-1} \circ R_{y} \circ R_{x}, \\
T_{x}=L_{x}^{-1} \circ R_{x}
\end{gathered}
$$

for all $x, y \in Q$. The loop $Q$ is called automorphic if $\operatorname{Inn}(Q)$ acts on $Q$ by automorphisms, and left automorphic if $\operatorname{LInn}(Q)$ does.

A loop $Q$ is automorphic if the mappings $\ell_{x, y}, r_{x, y}$ and $T_{x}$ for all $x, y \in Q$ are automorphisms of $Q$. A loop $Q$ is left automorphic if the mappings $\ell_{x, y}$ are automorphisms of $Q$. It is known for a Moufang loop $Q$ that if $\ell_{x, y}$ is an automorphism for some $x, y \in Q$ then $r_{x, y}$ is also an automorphism of $Q$ and vice versa (see [1]).

The property of a loop to be left automorphic is of significance in differential geometry. In particular, a reductive homogeneous space is completely determined by a loop with the property of left poweralternativity (or left monoalternativity) and the left automorphic property $([11],[17])$.

Each alternative (in particular, each diassociative) automorphic loop is Moufang ([12]). Also, each power-alternative left automorphic smooth loop is Moufang ([2]). Besides every smooth automorphic Moufang 
loop is a group. A well known class of non-trivial finite examples of left automorphic Moufang loops is provided by Code loops ([7]).

As usual one defines a normal subloop as the kernel of a loop homomorphism. A subloop of a given loop $Q$ is normal if and only if it is invariant under the $\operatorname{Inn}(Q)$.

Moufang loops are diassociative, that is the subloop generated by two elements is a group. Hence in a Moufang loop one has

$$
x \backslash y=x^{-1} y, y / x=y x^{-1} .
$$

In a Moufang loop $Q$ the commutator subloop $[Q, Q]$ is the subloop generated by all elements of the form $x^{-1} y^{-1} x y=[x, y]$, where $x, y \in Q$ and the associator subloop $(Q, Q, Q)$ is the subloop generated by all elements of the form $(x(y z))^{-1}((x y) z)=(x, y, z)$ for all $x, y, z \in Q$.

The subloop $Z(Q)=\{x \in Q:[x, a]=1, \forall a \in Q\}$ is called the commutant of $Q$.

The subloops

$$
\begin{aligned}
& N_{\lambda}(Q)=\{x \in Q:(x, a, b)=1, \forall a, b \in Q\}, \\
& N_{\mu}(Q)=\{x \in Q:(a, x, b)=1, \forall a, b \in Q\}, \\
& N_{\rho}(Q)=\{x \in Q:(a, b, x)=1, \forall a, b \in Q\}
\end{aligned}
$$

are called left, middle and right nucleus of $Q$. The subloop $N(Q)=$ $N_{\lambda}(Q) \cap N_{\mu}(Q) \cap N_{\rho}(Q)$ is called the nucleus of $Q$. In a Moufang loop $L$ all nuclei coincide. The subloop $C(Q)=Z(Q) \cap N(Q)$ is called the center of the loop $Q$.

All characteristic subloops of any automorphic loop are normal. In particular, $[Q, Q],(Q, Q, Q), Z(Q), N(Q), C(Q)$ are normal subloops of an automorphic Moufang loop $Q$.

\section{Main Theorem}

The aim of this note is to show the following

Theorem 3.1. Let $L$ be a finite automorphic Moufang loop and let $\tau$ be a half-automorphism of $L$. Then $\tau$ is an automorphism of $L$ or $\tau$ is an anti-automorphism of $L$.

Conjecture 3.2. For every automorphic Moufang loop all half-isomorphisms are trivial.

In order to prove this Theorem we need to collect some results.

We will use the notation $[u, v, w]=[[u, v] w]$ and by induction

$$
\left[a_{1}, \ldots, a_{n+1}\right]=\left[\left[a_{1}, \ldots, a_{n}\right], a_{n+1}\right]
$$


for left-normed commutators.

Lemma 3.3. (Bruck) Let $L$ be a left automorphic Moufang loop and assume $t, u, v \in L$. Then the following statements hold:

(i) If $L$ is generated by 3 elements, then the associator subloop $(L, L, L)$ lies in the center of $L$.

(ii) $[u, v] \in N(L)$.

(iii) $[u v, t]=[u, t][u, t, v][v, t]$,

(iv) $(n u, v, t)=(u, n v, t)=(u, v, n t)=(u, v, t)$ for all $n \in N(L)$.

(v) If $L$ is an automorphic Moufang loop, then $u^{3} \in N(L)$.

Proof. [1], chapter VII, Lemma 2.2 q.e.d.

Quite recently in the theory of finite Moufang loops a breakthrough has been reached: the Theorem of Lagrange and an important part of the Theorems of Sylow have been proved (see [9], [4], [10], [3]). In the following Lemma 3.4 and Lemma 3.5 these facts are used.

Lemma 3.4. Let $L$ be a finite automorphic Moufang loop, let $\tau$ be a half-automorphism of $L$ and let $S$ be a 3 -Sylow subloop of $L$. Then

(i) $L=S N(L)=N(L) S$,

(ii) $\tau(L, L, L)=(\tau(L), \tau(L), \tau(L))$,

(iii) $\tau(N(L))=N(\tau(L))$,

(iv) $\tau(C(L))=C(\tau(L))$,

(v) $\tau[L, L]=[\tau(L), \tau(L)]$.

Proof. It is known by (v) from the previous Lemma 3.3 that any finite automorphic Moufang loop $L$ is the product of $S$ and the nucleus $N(L)$ of $L$. Thus (i) holds.

Obviously $\tau(S)$ is a 3-Sylow subloop of $\tau(L)$. Applying (i) to $\tau(L)$ we obtain

$$
\tau(L)=\tau(S) \tau(N(L))=\tau(S) N(\tau(L)) .
$$

For arbitrary elements $u, v, w \in L$ we put $u=s_{1} n_{1}, v=s_{2} n_{2}, w=$ $s_{3} n_{3}$ with $s_{i} \in S, n_{i} \in N(L), 1 \leq i \leq 3$. Hence $(u, v, w)=\left(s_{1}, s_{2}, s_{3}\right)$ according to Lemma 3.3, (iv). In [5] it was shown that for Moufang loop of odd order any half-automorphism is trivial, so $\tau$ is trivial on $S$. If $\tau$ is an isomorphism then

$$
\tau(u, v, w)=\tau\left(s_{1}, s_{2}, s_{3}\right)=\left(\tau\left(s_{1}\right), \tau\left(s_{2}\right), \tau\left(s_{3}\right)\right)=(\tau(u), \tau(v), \tau(w)) .
$$

Otherwise straightforward computation shows that

$$
\tau(u, v, w)=\left(\tau(u)^{-1}, \tau(v)^{-1}, \tau(w)^{-1}\right)^{-1} .
$$


Hence in both cases we have

$$
\tau(L, L, L)=(\tau(L), \tau(L), \tau(L)) \text { and } \tau(N(L))=N(\tau(L))
$$

by the definition of $N(L)$.

It is evident that $\tau(Z(L))=Z(\tau(L))$ and $\tau(C(L)=C(\tau(L))$.

Finally, $\tau[L, L]=[\tau L, \tau L]$ since Moufang loops are diassociative. q.e.d.

In the Theory of Loops there exist different notions of nilpotency ([1],14] and [13]) which for groups, but not for loops in general, are equivalent. Here we will use the following definition:

A Moufang loop $M$ is commutatively nilpotent if for some $n \in \mathbb{N}$ any left-normed commutator word of length $n$ is trivial on $M$.

Lemma 3.5. Let $L$ be a finite commutatively nilpotent automorphic Moufang loop. Then

(i) $L$ is the direct product of its 3-Sylow subloop and its 3'-Hall subgroup, which is contained in the nucleus of $L$.

(ii) $L$ is the direct product of its Sylow subloops.

Proof. First we show (i). By Lemma 3.4 for every finite automorphic Moufang loop one has $L=S N(L)$, where $S$ is a 3-Sylow subloop of $L$. Let $a \in S$ and $b \in L$ such that the orders of $a$ and $b$ are coprime. The elements $a$ and $b$ generate a nilpotent subgroup of $L$. Thus they commute. On the other hand, $N(L)$ is a nilpotent subgroup of $L$. Hence $N(L)$ is the direct product of its Sylow subgroups. Let $\hat{N}$ be the direct product of all Sylow subgroups of $N(L)$ apart of its 3-Sylow subgroup. Then $L=S \hat{N}$ and $S \cap \hat{N}=1$. Since $\hat{N}$ is subgroup of $N(L)$ it follows immediately that every element $a \in L$ has a unique presentation in the product $S \hat{N}$. Indeed $s_{1} n_{1}=s_{2} n_{2}$ for $n_{1}, n_{2} \in \hat{N}$, and $s_{1}, s_{2} \in S$ implies $s_{2}^{-1} s_{1}=n_{2} n_{1}^{-1}$.

Using again the fact that $\hat{N} \leq N(L)$ by straightforward verification one can show that $S$ is invariant under the inner mappings group $\operatorname{Inn}(L)$ and $\hat{N}$ is also invariant under the inner mappings group $\operatorname{Inn}(L)$. For example,

$$
l_{a, b}(s)=(a b)^{-1}(a(b(s)))=\left(s_{1} n_{1} \cdot s_{2} n_{2}\right)^{-1}\left(s_{1} n_{1}\left(s_{2} n_{2}(s)\right)\right)=s_{3},
$$

where $s, s_{1}, s_{2}, s_{3} \in S, n_{1}, n_{2} \in \hat{N}$.

It follows that $L$ is the direct product of its 3-Sylow subloop and its 3 - Hall subgroup, which is contained in the nucleus of $L$. By analogous arguments one can show that every Sylow subloop is normal in $L$, and thus we have the second statement of the Lemma (see [1], p.72). q.e.d. By Lemma 3.4,(ii) one can define the induced half-isomorphism

$$
\bar{\tau}: L /(L, L, L) \rightarrow \tau(L) /(\tau(L), \tau(L), \tau(L))
$$


on a finite automorphic Moufang loop $L$ with a half-isomorphism $\tau$. Denote $\bar{L}=L /(L, L, L)$ and $\tau \overline{(L)}=\tau(L) /(\tau(L), \tau(L), \tau(L))$

Lemma 3.6. Let $L$ be a finite automorphic Moufang loop with a nontrivial half-isomorphism $\tau$. Consider the induced half-isomorphism

$$
\bar{\tau}: \bar{L} \rightarrow \tau(\bar{L})
$$

Then the following statements hold:

(i) $\bar{\tau}$ is a trivial half-isomorphism.

(ii) If $L$ is generated by 3 elements and if $\bar{\tau}$ is an isomorphism on the group $\bar{L}$, then for every pair $u, v \in L$ satisfying the condition

$$
\tau(u v)=\tau(v) \tau(u)
$$

one has $[u, v] \in C(L)$ and $[\tau(u), \tau(v)] \in C(\tau(L))$.

(iii) If $L$ is generated by 3 elements and if $\bar{\tau}$ is an anti-isomorphism, then $[w, t] \in C(L)$ and $[\tau(w), \tau(t)] \in C(\tau(L))$ for all $w, t \in L$ satisfying the condition

$$
\tau(w t)=\tau(w) \tau(t)
$$

Proof. (i) Since $L /(L, L, L)$ is a group it follows from Scott's Theorem [18] that $\bar{\tau}$ is an isomorphism or an anti-isomorphism.

(ii)Assume first that $\bar{\tau}$ is an isomorphism. For $u, v \in L$ and $\bar{u}=$ $u(L, L, L)$,

$\bar{v}=v(L, L, L)$ we get $\bar{\tau}(\bar{u} \bar{v})=\bar{\tau}(\bar{u}) \bar{\tau}(\bar{v})$. We have on the other hand $\bar{\tau}(\bar{u} \bar{v})=\bar{\tau}(\bar{v}) \bar{\tau}(\bar{u})$. Thus the commutator $[\bar{\tau}(\bar{u}), \bar{\tau}(\bar{v})]$ is trivial, and $[\tau(u), \tau(v)]$ is in the $(\tau(L), \tau(L), \tau(L))=\tau(L, L, L)$ and consequently by Lemma 3.3 , (i) one knows that $[\tau(u), \tau(v)]$ is in the center of $\tau(L)$. Using half-isomorphism $(\tau)^{-1}$ analogously one can show that $[u, v] \in$ $C(L)$. Hence statement (ii) is shown.

As we mentioned above every anti-isomorphism is the composition of some isomorphism and inverse mapping, so one can treat the case (iii) of an anti-isomorphism $\bar{\tau}$ in a similar way.

q.e.d.

In what follows we will consider the case that $\bar{\tau}$ is an isomorphism on $\bar{L}$. If $\bar{\tau}$ is an anti-isomorphism on $\bar{L}$ one can prove the Theorem 3.1 using the inverse mapping and analogous arguments.

Lemma 3.7. Let $L$ be a finite automorphic Moufang loop generated by 3 elements with a nontrivial half-isomorphism $\tau$. Put

$$
D(L, \tau)=\{g \in L \mid \exists h \in L: \tau(g h)=\tau(h) \tau(g) \neq \tau(g) \tau(h)\} .
$$

If $d \in[L, L]$, then $[d, D(L, \tau)] \subseteq C(L)$. 
Proof. Let $d \in[L, L], g \in D(L, \tau)$, choose $h \in L$ such that $\tau(g h)=$ $\tau(h) \tau(g) \neq \tau(g) \tau(h)$. Since $[L, L] \subseteq N(L)$ a subloop $L_{0}$ generated by $d, h, g$ is a group. By Scott's Theorem $\tau$ restricted to $L_{0}$ is an antimonomorphism. Hence $\tau(d g)=\tau(g) \tau(d)$. If $\tau(g) \tau(d) \neq \tau(d) \tau(g)$, then by Lemma 3.6 we get $[g, d] \in C(L)$. Finally, if $\tau(g) \tau(d)=\tau(d) \tau(g)$, then $[g, d]=1$.

q.e.d.

Lemma 3.8. (Gagola-Giuliani [5]) Let $M$ be a finite Moufang loop. Let $\tau$ be a half-isomorphism of $M$. Then

(i) $\tau$ is a semi-isomorphism of $M$, i.e. for any $u, v \in M \tau(u v u)=$ $\tau(u) \tau(v) \tau(v)$

(ii) If $M$ is of odd order, then $\tau$ is trivial. In this case in particular $\tau(M)$ is isomorphic to $M$.

(iii) If $\tau$ is not trivial, then there exist $x, y, z \in M$ such that $[x, y] \neq 1$, $[x, z] \neq 1$ and

$$
\begin{aligned}
& \tau(x y)=\tau(x) \tau(y) \neq \tau(y) \tau(x), \\
& \tau(x z)=\tau(z) \tau(x) \neq \tau(x) \tau(z) .
\end{aligned}
$$

q.e.d.

Let us call such elements $\{x, y, z\}$ as it is described above in the Lemma 3.8 a Gagola-Giuliani triple or $G G$-triple for short.

Lemma 3.9. Let $L$ be a finite automorphic Moufang loop with a nontrivial half-automorphism $\tau$. A subloop of $L$ generated by a GagolaGiuliani triple is commutatively nilpotent.

Proof. Let $L$ be a finite automorphic Moufang loop with non-trivial half-automorphism $\tau$ and let $\{x, y, z\}$ be a $G G$-triple of a loop $L$. Let us recall that this means that conditions (iii) of Lemma 3.8 hold. Hence (1) implies that $\left.\tau\right|_{\langle x, y\rangle}$ is a monomorphism and (2) implies that $\left.\tau\right|_{\langle x, z\rangle}$ is an anti-monomorphism. Thus there are two possibilities for the subgroup $\langle y, z\rangle$ : either $\left.\tau\right|_{\langle y, z\rangle}$ is a monomorphism or $\left.\tau\right|_{\langle y, z\rangle}$ is an antimonomorphism.

Put $\tau(x)=a, \tau(y)=b, \tau(z)=c$. Note that $\{a, b, c\}$ is a $G G$-triple if and only if $\{x, y, z\}$ is a $G G$-triple. Indeed the conditions (1) and (2) of Lemma 3.8 are equivalent to

$$
\begin{aligned}
& \tau^{-1}(a b)=\tau^{-1}(a) \tau^{-1}(b) \neq \tau^{-1}(b) \tau^{-1}(a) \\
& \tau^{-1}(a c)=\tau^{-1}(c) \tau^{-1}(a) \neq \tau^{-1}(a) \tau^{-1}(c)
\end{aligned}
$$


Let $L_{1}$ be a loop generated $x, y, z$ and $L_{2}$ be a loop generated by $a, b, c$. Obviosly the mappings $\left.\tau\right|_{L_{1}}: L_{1} \rightarrow L_{2}$ and $\left.\tau^{-1}\right|_{L_{2}}: L_{2} \rightarrow L_{1}$ are nontrivial half-isomorphisms.

Case 1: Suppose that $\left.\tau\right|_{\langle y, z\rangle}$ is an anti- monomorphism and

$$
\tau(y z)=\tau(z) \tau(y)=c b \neq \tau(y) \tau(z)=b c .
$$

One has $\left[L_{2}, L_{2}, u\right] \subseteq C\left(L_{2}\right)$, where $u \in\{a, b, c\} \subseteq D\left(L_{2}, \tau^{-1}\right)$ by Lemma 3.7. Hence $\left[L_{2}, L_{2}, L_{2}\right] \subseteq C\left(L_{2}\right)$ by Lemma 3.3, (iii) and $L_{2}$ is commutatively nilpotent of class $\leq 4$.

Case 2: Suppose that $\left.\tau\right|_{\langle y, z\rangle}$ is a monomorphism. Let us study the behavior of the half-isomorphism $\tau$ restricted to the following twogenerated subgroup:

$$
L_{3}=\langle x y, z x\rangle,
$$

Suppose that $\tau$ restricted to $L_{3}$ is a monomorphism. Then

$$
\tau(x y \cdot z x)=\tau(x y) \tau(z x)=a b \cdot a c .
$$

By the Moufang identity and by Lemma 3.8(i),

$$
\tau(x y \cdot z x)=\tau(x \cdot y z \cdot x)=a \cdot b c \cdot a=a b \cdot c a .
$$

Hence $a c=c a$ which contradicts the condition that $\{x, y, z\}$ is GGtriple. Therefore $\tau$ restricted to $L_{3}$ is an anti-monomorphism. Now by Lemma 3.6 one has $[a b, a c] \in C\left(L_{2}\right)$ as well as $[a, c] \in C\left(L_{2}\right)$. But it can also be proven that

$$
[a b, a c]=[a, c] \neq 1 .
$$

In order to see this, note that

$$
\begin{aligned}
a c \cdot a b & =\tau(z x) \tau(x y)=\tau(x y \cdot z x) \\
& =\tau(x \cdot y z \cdot x)=a \cdot b c \cdot a=a b \cdot c a,
\end{aligned}
$$

Let us consider the $G G$-triple $\{a, a b, a c\}$. The map $\tau^{-1}$ restricted to $\langle a, a b\rangle$ is a monomorphism, $\tau^{-1}$ restricted to $\langle a, a c\rangle$ is an antimonomorphism, and $\tau^{-1}$ restricted to $\langle a b, a c\rangle$ is an anti-monomorphism too. Thus we have the conditions of the Case 1 and therefore the loop generated by $\{a, a b, a c\}$ is commutatively nilpotent. But

$$
L_{2}=\langle a, a b, a c\rangle=\langle a, b, c\rangle .
$$

The Lemma is proved.

q.e.d.

Now everything is ready to prove the Theorem 3.1 .

By Lemma 3.5 the loop $L_{2}$ is a direct product of its Sylow subloops. So $\tau^{-1}$ acts componentwise on $L_{2}$. All Sylow subloops except the 3Sylow subloop are groups, and the 3 -Sylow subloop is a loop of odd 
order. By the Lemma 3.8(ii) $\tau^{-1}$ is a trivial half-isomorphism on $L_{2}$, which forms a contradiction with the conditions of Lemma 3.9. Thus Theorem 3.1 is proved.

q.e.d.

\section{EXAMPLES.}

We give two examples of loops which admit a non-trivial half-automorphism. Both examples are in a different way not too far away from being automorphic Moufang loops. All our statements about these examples are easily checked using the LOOPS package of GAP [15]

1. Let $Q_{1}$ be the loop of order 16 given by the Cayley table

\begin{tabular}{|c||c|c|c|c|c|c|c|c|c|c|c|c|c|c|c|c|}
\hline$\circ$ & 1 & 2 & 3 & 4 & 5 & 6 & 7 & 8 & 9 & 10 & 11 & 12 & 13 & 14 & 15 & 16 \\
\hline \hline 1 & 1 & 2 & 3 & 4 & 5 & 6 & 7 & 8 & 9 & 10 & 11 & 12 & 13 & 14 & 15 & 16 \\
\hline 2 & 2 & 4 & 8 & 6 & 3 & 1 & 5 & 7 & 14 & 9 & 16 & 10 & 11 & 12 & 13 & 15 \\
\hline 3 & 3 & 5 & 4 & 7 & 6 & 8 & 1 & 2 & 15 & 13 & 9 & 11 & 14 & 16 & 12 & 10 \\
\hline 4 & 4 & 6 & 7 & 1 & 8 & 2 & 3 & 5 & 12 & 14 & 15 & 9 & 16 & 10 & 11 & 13 \\
\hline 5 & 5 & 7 & 2 & 8 & 4 & 3 & 6 & 1 & 13 & 11 & 14 & 16 & 12 & 15 & 10 & 9 \\
\hline 6 & 6 & 1 & 5 & 2 & 7 & 4 & 8 & 3 & 10 & 12 & 13 & 14 & 15 & 9 & 16 & 11 \\
\hline 7 & 7 & 8 & 1 & 3 & 2 & 5 & 4 & 6 & 11 & 16 & 12 & 15 & 10 & 13 & 9 & 14 \\
\hline 8 & 8 & 3 & 6 & 5 & 1 & 7 & 2 & 4 & 16 & 15 & 10 & 13 & 9 & 11 & 14 & 12 \\
\hline 9 & 9 & 10 & 11 & 12 & 16 & 14 & 15 & 13 & 4 & 6 & 7 & 1 & 5 & 2 & 3 & 8 \\
\hline 10 & 10 & 12 & 16 & 14 & 15 & 9 & 13 & 11 & 2 & 4 & 5 & 6 & 3 & 1 & 8 & 7 \\
\hline 11 & 11 & 13 & 12 & 15 & 10 & 16 & 9 & 14 & 3 & 8 & 4 & 7 & 6 & 5 & 1 & 2 \\
\hline 12 & 12 & 14 & 15 & 9 & 13 & 10 & 11 & 16 & 1 & 2 & 3 & 4 & 8 & 6 & 7 & 5 \\
\hline 13 & 13 & 15 & 10 & 16 & 9 & 11 & 14 & 12 & 8 & 7 & 2 & 5 & 4 & 3 & 6 & 1 \\
\hline 14 & 14 & 9 & 13 & 10 & 11 & 12 & 16 & 15 & 6 & 1 & 8 & 2 & 7 & 4 & 5 & 3 \\
\hline 15 & 15 & 16 & 9 & 11 & 14 & 13 & 12 & 10 & 7 & 5 & 1 & 3 & 2 & 8 & 4 & 6 \\
\hline 16 & 16 & 11 & 14 & 13 & 12 & 15 & 10 & 9 & 5 & 3 & 6 & 8 & 1 & 7 & 2 & 4 \\
\hline
\end{tabular}

Then $Q_{1}$ is a Code loop (see [7]) in particular it is a left automorphic Moufang loop. One can see that the mapping $\phi: Q_{1} \rightarrow Q_{1}$ defined by

$$
\phi(5)=8, \phi(8)=5, \phi(x)=x \text { for } x \notin\{5,8\}
$$

is a half-automorphism. Since

$$
\begin{aligned}
& \phi(2 \circ 7)=\phi(5)=8=\phi(7) \circ \phi(2) \neq \phi(2) \circ \phi(7)=5 \\
& \phi(3 \circ 9)=\phi(15)=15=\phi(3) \circ \phi(9) \neq \phi(9) \circ \phi(3)=11
\end{aligned}
$$

this half-automorphism is non-trivial.

2. The loop $Q_{2}$ defined by the Cayley table 


\begin{tabular}{|l||l|l|l|l|l|l|l|l|}
\hline$\circ$ & 1 & 2 & 3 & 4 & 5 & 6 & 7 & 8 \\
\hline \hline 1 & 1 & 2 & 3 & 4 & 5 & 6 & 7 & 8 \\
\hline 2 & 2 & 1 & 4 & 3 & 6 & 5 & 8 & 7 \\
\hline 3 & 3 & 4 & 1 & 2 & 7 & 8 & 6 & 5 \\
\hline 4 & 4 & 3 & 2 & 1 & 8 & 7 & 5 & 6 \\
\hline 5 & 5 & 6 & 8 & 7 & 1 & 2 & 4 & 3 \\
\hline 6 & 6 & 5 & 7 & 8 & 2 & 1 & 3 & 4 \\
\hline 7 & 7 & 8 & 5 & 6 & 3 & 4 & 2 & 1 \\
\hline 8 & 8 & 7 & 6 & 5 & 4 & 3 & 1 & 2 \\
\hline
\end{tabular}

is an automorphic loop, but not a Moufang loop and the permutation $\phi=(3,5)(4,6)(7,8)$ is a half-automorphism of $Q_{2}$. By

$\phi(4 \circ 6)=\phi(7)=8=\phi(4) \circ \phi(6) \neq \phi(6) \circ \phi(4)=7$

$\phi(4 \circ 8)=\phi(6)=4=\phi(8) \circ \phi(4) \neq \phi(4) \circ \phi(8)=3$

$\phi$ is no-trivial half-automorphism.

Acknowledgements. The authors thank to Stephen Gagola III, Jacob Mostovoy and Peter Plaumann for discussions about some details of the article and to Alberto Elduque for his help to improve the article. The first author thanks to FAPESP, CNPQ and russian grant RFFI 13-01-00239. The second author thanks to SRE, Mexico to support her visit to UAEM, Mexico during May-July, 2012. The third author thanks to the russian grant 2014/319. The fourth author thanks for support to 2011-2013 UCMEXUS-CONACYT Collaborative Grant CN-11-567,

2012-2013 FAPESP Grant processo 2012/11068-2 and

2012-2013 CONACYT Grant for Sabbatical year at the Institute of Mathematics and Statistics of the University of Sao Paulo, Brazil.

\section{REFERENCES}

[1] R. Bruck, A Survey of Binary Systems, Springer-Verlag (1966)

[2] R. Carrillo Catalan, L. Sabinina, On smooth power alternative loops, Communications in Algebra 32, no. 8 (2004), 2969-2976.

[3] S. Gagola III, The development of Sylow $p$-subloops in finite Moufang loops, J. Algebra, 322, no. 8 (2009), 2804-2810.

[4] S. Gagola III, J.I. Hall, Lagrange's theorem for Moufang loops, Acta Sci. Math. (Szeged) 71 (2005), no. 1-2 (2005), 45-64.

[5] S. Gagola III, M.L. Merlini Giuliani, Half-isomorphism of Moufang loops of odd order, J. of Alg. and Appl, 11, no. 5 (2012), 194-199.

[6] S. Gagola III, M.L. Merlini Giuliani, On half-automorphisms of certain Moufang loops with even order, J. of Algebra, 386 (2013), 131-141.

[7] R.I. Griess, Code loops. J.of Algebra. 100, no. 1 (1986), 224-234. 
[8] A. Grishrov, P. Plaumann L. Sabinina, Structure of free automorphic loops. Proc. Amer. Math. Soc. 140, no. 7 (2012), 2209- 2214.

[9] A. Grishkov, A. Zavarnitsine, Lagrange's theorem for Moufang loops, Math. Proc. Cambridge Philos. Soc. 139, no. 1 (2005), 41-57.

[10] A. Grishkov, A. Zavarnitsine, Sylow's theorem for Moufang loops. J.of Algebra 321, no. 7 (2009), 1813-1825.

[11] M. KikKawa, Geometry of homogeneous Lie loops, Hiroshima Math J. 5 (1975), 141-179

[12] M. Kinyon, K. Kunen,J. D. Phillips, Every diassociative $A$ - loop is Moufang. Proc. Amer. Math. Soc. 130 (2004), 619-624 .

[13] J. Mostovoy, Nilpotency and Dimension Filtration for Loops, Comm. Algebra 36 , no. 4 (2008), 1565-1579.

[14] J. Mostovoy, J.M. Perez-Izquierdo, Dimension Filtration on Loops, Israel J. Math. 158 (2007), 105-118.

[15] G. Nagy, P. Vojtechovsky, LOOPS.Version 2.2.0. Computing with quasigroups and loops in GAP. preprint

[16] P. Plaumann, L. Sabinina, On nuclearly nilpotent loops of finite exponent. Comm. in Algebra 36 (2008), 1346-1353.

[17] L. Sabinin, Smooth quasigroups and loops. Kluwer (1999).

[18] W. R. ScOtт, Half-Homomorphisms of Groups, Proc. of the AMS 8, no. 6 (1957), 1141-1144.

A.Grishkov, Instituto de Matemática e Estatística, Universidade de São Paulo, Rua de Matão 1010, São Paulo, 05508-090, Brazil

E-mail address: grishkov@ime.usp.br

M.L.Merlini Giuliani, Universidade Federal do ABC, Av. dos Estados, 5001 Bairro Bangu, Santo André SP 09210-580 - Brazil,

E-mail address: maria.giuliani@ufabc.edu.br

M. Rasskazova, Omsk State Institute of Service, Pevtsova street 13, Omsk, 644099, Russia

E-mail address: marinarasskazova@yandex.ru

L. Sabinina, Universidad Autónoma del Estado de Morelos, Av. UniVersidad 1001, Cuernavaca, 62209, MÉXico

E-mail address: liudmila@uaem.mx 